\title{
Response inhibition of rats with medial and lateral septal lesions
}

RUSSELL L. AGNEW and MERLE E. MEYER, Western Washington State College, Bellingham, Wash. 98225

Ten male albino rats were shaped to bar press for food pellets and placed on a differential reinforcement of low-rate $(D R L)$ schedule to investigate the effects of lesions in medial septal rats and lateral septal rats on response inhibition. All Ss in both experimental groups, including those that failed to meet the 50\% response reinforcement criterion, did show a gradual and distinct acquisition curve. For all groups, as training progressed, an attenuation in response rate was accompanied by an increase in number of reinforcements. Mann-Whitney $U$ tests indicate that the differences between the control group and each experimental group were significant at the .05 level. No significant differences were found between the medial septal group and the lateral septal group.

Several investigations have attempted to determine the effects of septal lesions upon bar-pressing behavior of rats on a temporal discrimination schedule for food reinforcement. Two factors of interest are the loci of ablation, and the various differential reinforcement of low rate (DRL) schedules employed by investigators. Ellen, Wilson, \& Powell (1964) found that rats in which only the medial septal area had been ablated showed impairment in acquisition of DRL performance. On the other hand, Caplan \& Stamm (1967) found that acquisition of DRL behavior in animals with septal lesions in both the medial and lateral regions depend upon the training method employed, and the specific delay interval required for reinforcement. Septal rats placed directly on a DRL-20, the abrupt method, failed to inhibit timing behavior, whereas septals that were placed on a DRL-6 schedule did learn to inhibit their responses. If the DRL contingency is lengthened by small discrete steps as the animal improves his timing behavior at each preceding one, it will acquire a DRL-20 (Caplan \& Stamm, 1967). Further, Harvey \& Hunt (1965) reported that medial septals performed at least as well as normals when working for water on a gradually increasing DRL contingency.

The present study was conducted to determine the extent to which each septal region, medial or lateral, disrupts acquisition of response inhibition (timing behavior) as measured in terms of differential reinforcement of low-rate performance in the rat.

\section{SUBJECTS}

Twelve male Sprague-Dawley rats, 120 days old, were obtained from the Rush Laboratories. Four rats were randomly assigned to the control group, four to the medial septal group, and four to the lateral septal group. One rat assigned to the lateral septal group expired while being anesthetized for the operation and was immediately replaced by one of the reserves; another rat was dropped from the medial group after histological examination.

\section{APPARATUS}

The apparatus consisted of two conventional operant boxes, each placed in a sound deadened chamber equipped with a circulating fan and white noise, which served as an auditory mask. Standard relay equipment was used to program all reinforcement contingencies. The reinforcers were $45-\mathrm{mg}$ Noyes pellets, and were given under the contingency of a DRL 15-sec schedule. The responses and reinforcements were recorded on counters.

\section{PROCEDURE}

Following a 23-h food-deprivation period all Ss were initially shaped to bar press for a food pellet. They were then placed on a continuous reinforcement schedule for $1 \mathrm{~h}$. Subsequent to preliminary training, each $\mathbf{S}$ assigned to the medial or lateral septal group was administered an ether anesthetic and placed in the Stellar-type stereotaxic apparatus. The de Groot (1959) Brain Atlas was used for reference coordinates. Lesions were made bilaterally in the predetermined area of the septum following the standard procedures. For the lateral and medial septal lesions, a direct current of $3 \mathrm{~mA}$ was administered for $15 \mathrm{sec}$. The medial septal lesions were placed $1.9 \mathrm{~mm}$ in front of Bregma, $\pm .5 \mathrm{~mm}$ lateral to the sagittal suture, and $6.5 \mathrm{~mm}$ below the surface of the skull at this point. The lateral septal lesions were placed $2.3 \mathrm{~mm}$ in front of Bregma, $\pm 1.0 \mathrm{~mm}$ lateral to the sagittal suture, and $5.0 \mathrm{~mm}$ below the surface at this point. The control rats were subjected to similar operative procedures, but did not receive any electric current. After the operation the Ss were placed in metabolism cages on food and water ad lib for a recuperation period of 7 days.

One day prior to the initiation of the DRL schedule all Ss were again subjected to $23 \mathrm{~h}$ food deprivation, after which each $S$ was placed in the operant chamber on the DRL 15 -sec schedule. Each $S$ continued on that schedule until reaching the final criterion performance of three consecutive 50 -min daily training periods in which the $S$
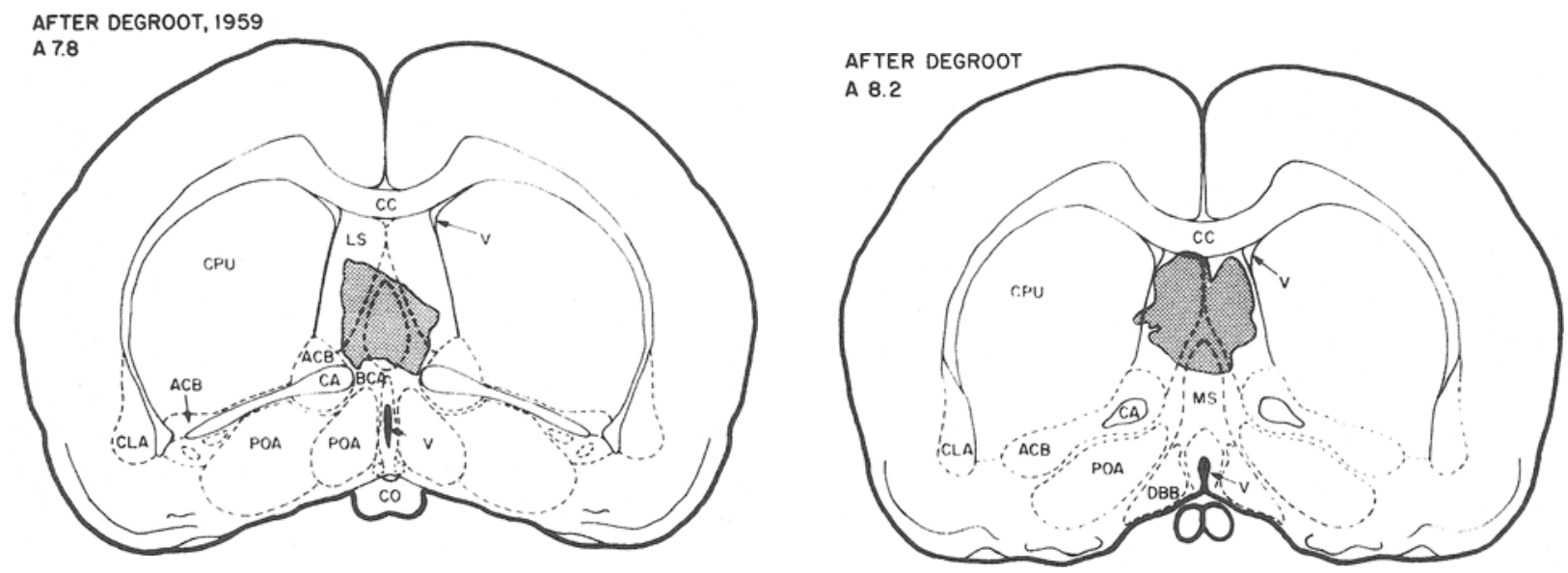

Fig. 1. Reconstruction of a medial septal lesion (left) for S 6 and a lateral septal lesion (right) for $\mathbf{S} 7$. 

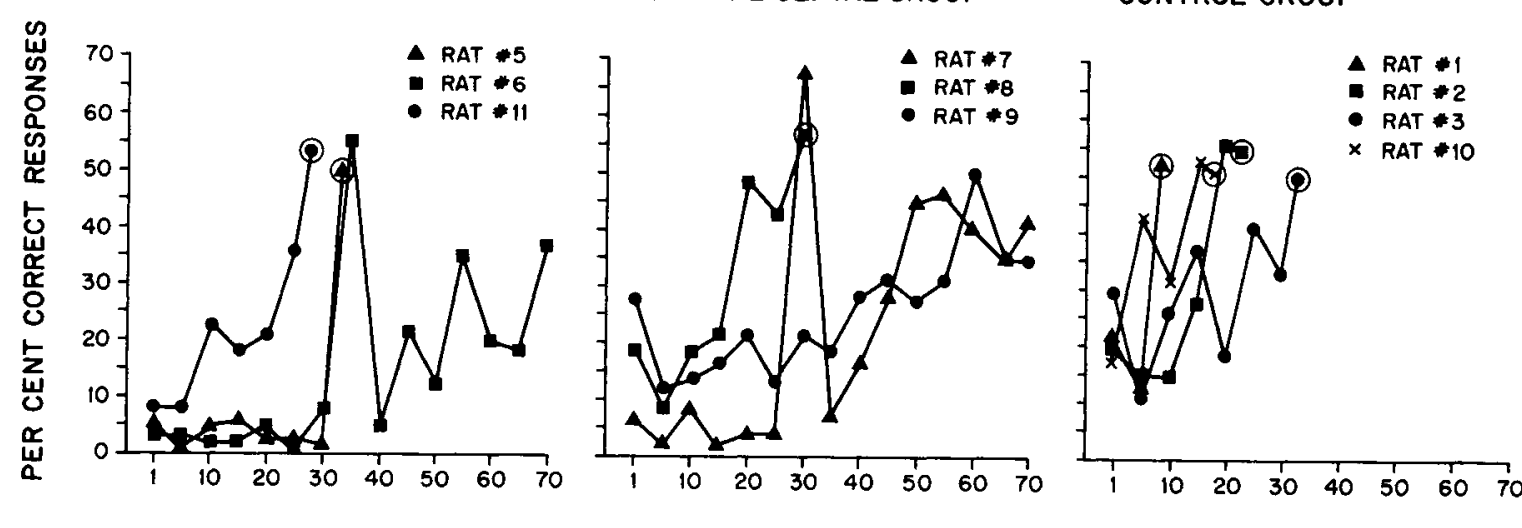

DAYS TO CRITERION

Fig. 2. Acquisition of DRL behavior by the medial septal, lateral septal, and control groups. Criterion is denoted by the circle around respective rat symbols.

received reinforcements on at least $50 \%$ of the responses, or until 70 days had elapsed.

As each $S$ attained criterion, it was sacrificed, perfused with $10 \%$ formalin, and had its brain removed for histological examination. If a $S$ had not reached criterion by the 70th consecutive day, it was also sacrificed and put through the same procedure.

\section{RESULTS}

Anatomical Findings

All medial and lateral septal lesions were verified histologically. The lesions as presented in Fig. 1 represent coronal sections of the brain for S 6 and S 7. The medial septal group sustained extensive bilateral damage to the medial septal nuclei and slight damage anteriorly to the diagonal band of Broca, anterior commissure, lateral parolfactorial area, lateral septal nuclei, posteriorly to the bed nucleus, hippocampal commissure, triangular septal nucleus, and the postcommissural columns of the fornix. The rank order from the least to the greatest extent was 11,5 , and 6 . The lateral septal group sustained extensive bilateral damage to the lateral septal nuclei and slight damage anteriorly from the area of the medial septal nucleus and diagonal band of Broca ventrally to the corpus collosum dorsally, the Horns of Ammon, and unilaterally to the caudate putamen. Posteriorly, damage was limited to the bed nucleus, hippocampal commissure, the triangular septal nucleus, and the columns of fornix. The rank order from the least to the greatest extent was 8,9 , and 7.

Behavioral Findings

All lesioned Ss, including those animals that failed to meet the $50 \%$ criterion, did show a gradual and distinct acquisition curve. It can be seen that two of the medial septal animals reached criterion in 28 and 34 days, respectively, while the third did not. One lateral septal rat reached criterion in $\mathbf{3 0}$ days, while the two remaining Ss did not. All controls acquired the DRL-15 schedule in the mean time of 22.25 days. Mann-Whitney $\mathrm{U}$ tests indicate the differences between the control group and each of the experimental groups were significant (both Us $=1, n_{1}=3$, $\mathrm{n}_{2}=4, \mathrm{p}=0.05$ ). No significant differences were found between the medial septal group and the lateral septal group $\left(\mathrm{U}^{\prime}=2.5\right.$, $\left.n_{1}=3, n_{2}=3, p=0.20\right)$.

On the first day of training a high rate of response was present in all groups of animals with relatively infrequent occurrence of reinforcement. As training progressed, the response rate decreased in all groups. For all groups this attenuation in response rate was accompanied by an increase in the number Whitney U-tests produced significant differences between the total number of responses on the first day and the last day $(p=0.05)$. Also, the increase in number of reinforcements on the last day's from that of the first day's session was significant $(p=0.05)$.

\section{DISCUSSION}

The data of the present study attempted to ascertain the extent to which lesions in the medial or lateral septal region would disrupt response inhibition as measured in terms of DRL performance. Contrary to the findings of several investigators (Ellen et al, 1964; Burkett \& Bunnell, 1966), severe impairment was not evident in the present of reinforcements. For each group, Mann-

study. Lesions limited to the septal area only impeded the acquisition of DRL performance as shown by the decreased rate of response and increased rate of reinforcement. A possible explanation may be the minute size of lesions sustained in the septal area. Others have reported considerably larger lesions that destroyed not only the intended area but disrupting pathways which may mediate the animal's ability to inhibit responses. It is evident from a number of studies (Brady \& Nauta, 1953; Fox, 1940; Powell, 1963) that septal nuclei receive connections from the olfactory bulb, frontal region, amygdala, olfactory tubercule, hippocampus, thalamus, and hypothalamus, etc. Distruction of these efferent pathways could very well effect the findings of impairment reported by other investigators. It should be pointed out that in the present study no successful lesion avoided some degree of invasion of the medial or lateral septal area. Ellen \& Powell (1962) found that small hippocampal lesions decrease the response rate under the fixed-interval schedule, indicating that the disturbance of the function of this structure by septal damage is not the critical factor in producing the terminal response rate increase. If the many seemingly contradictory studies in the literature were ordered on a continuum of lesion size, it might be found that the larger the lesion the greater the deficit in response inhibition.

Table 1

The Means for the Total Number of Responses and Reinforcements Obtained by Each Group of Animals on the First Day and the Last Day of the Acquisition Period

\begin{tabular}{lccccc} 
& \multicolumn{2}{c}{ First Day Mean Totals } & & \multicolumn{2}{c}{ Last Day Mean Totals } \\
\cline { 2 - 3 } \cline { 5 - 6 } Group & Responses & Reinforcements & & Responses & Reinforcements \\
\hline Controls & 366 & 54.50 & & 153.75 & 80.75 \\
Medial Septals & 572 & 24.67 & 191.33 & 48.67 \\
Lateral Septals & 617.67 & 36.67 & & 245.33 & 69.33 \\
\hline
\end{tabular}


Employment of the DRL 15-sec schedule by the abrupt method as defined by Caplan and Stamm did not prevent the septal animals from learning to inhibit reinforced responses. According to Ellen et al, if a bar-press response can be inhibited beyond $10 \mathrm{sec}$ then that is evidence of temporally dependent bar pressing. Such is the case in the present experiment. This suggests that a considerable part of the septal inhibitory influence is mediated through other parts of the limbic system as well. Earlier findings of Kimura (1958), Isaacson \& Wickelgren (1962), and Kimble (1963) seem to establish the fact that hippocampally lesioned animals show decreased ability to inhibit certain kinds of responses. But as of now, the literature involving the septal and hippocampal structure is inconclusive.

The findings of this study raise a question about the explanation of septal impairment in terms of a deficit in the ability to withhold a response (McCleary, 1961).

\section{REFERENCES}

BRADY, J. V. The paleocortex and behavioral motivation. WRAIR-30-56, Project 6-60-10-016, Subtask No. 7, January 1956.

BRADY, J. V., \& NAUTA, W. J. H. Subcortical mechanisms in emotional behavior: Affective changes following septal forebrain lesions in the albino rat. Journal of Comparative \& Physiological Psychology, 1953, 46, 339-346.

BURKETT, E. E., \& BUNNELL, B. N. Septal lesion and the retention of $D R L$ performance in the rat. Journal of Comparative \& Physiological Psychology, 1966, 62, 468-471.
CAPLAN, M., \& STAMM, J. DRL acquisition in rats with septal lesions. Psychonomic Science, $1967,8,5-6$.

de GROOT, J. The rat forebrain in stereotaxic coordinates. Verhandelingen Der Kaninklijke Nederlandse Akademie Van Wetenschappen, Afd. Natuurkunde, 1959, 2, 1.40.

ELLEN, P., \& POWELL, E. W. Temporal discrimination in rats with rhinencephalic lesions. Experimental Neurology, 1962,6, 538-547.

ELLEN, P., \& POWELL, E. W. Septal inhibition and timing behavior in the rat. Experimental Neurology, 1964, 10, 120-132.

FOX, C. A. Certain basal telencephalic centers. Journal of Comparative Neurology, 1940, 72, $1-62$.

HARVEY, J. A., \& HUNT, H. F. Effect of septal lesions on thirst in the rat as indicated by water consumption and operant responding for water reward. Journal of Comparative \& Physiological Psychology, 1965, 59, 49-56.

ISAACSON, R. L., \& WICKELGREN, W. O. Hippocampal ablation and passive avoidance. Science, 1962, 138, 1104-1106.

KIMBLE, D. P. The effects of bilateral hippocampal lesions in rats. Journal of Comparative \& Physiological Psychology, 1963, $55,661-670$.

KIMURA, D. Effects of selective hippocampal damage on avoidance behavior in the rat. Canadian Journal of Psychology, 1958, 12, 213-218.

MCCLEARY, R. A. Response specificity in the behavioral effects of limbic system lesions in the cat. Journal of Comparative \& Physiological Psychology, 1961, 54, 506-513.

POWELL, E. W. Septal efferents revealed by axonal degeneration in the rat. Experimental Neurology, 1963, 8, 406-422.

YOUNG, $M$. W. The nuclear pattern and fiber connections of the noncortical centers of the telencephalon of the rabbit (Lepus cuniculus). Journal of Comparative Neurology, 1936, 65 . 295-401. reduced when Ss were turned 360 deg once during the intertrial interval, and eight horizontal turns eliminated alternation behavior completely. Douglas assumed that horizontally tuming an $S$ acted to disrupt normal semicircular canal functioning.

\section{EXPERIMENT 1}

The primary purpose of this study was to investigate the effects of a 360-deg turn on subsequent choice-point behavior. One may consider the last quarter of a $\mathrm{CW}$ turn a 90-deg easterly/right turn when the start box is placed at the south end of the maze; a subsequent alternation response would be a westerly/left turn at the choice point. A second hypothesis to be tested concerned the very short-term memory of rats. If rats rely on a very short-term memory capacity in SAB, then moving the start box more proximal to the choice point should decrease the ITI and thereby increase the tendency to alternate.

\section{Method}

The Ss were 50 140-day-old male albino rats of the Sprague-Dawley strain purchased from Holtzman Company, Madison, Wis. Nineteen of the animals had served as Ss in a straight-runway study, $15 \mathrm{Ss}$ had been in a $T$-maze experiment, and 16 Ss were naive.

A single-unit, undifferentiated open-roofed T-maze was used. The dimensions of the T-maze were as follows: all alleys were $51 / 2$ in. wide and 14 in. high; goal alleys were 24 in. long; the start stem, and the start boxes located within the start stem, were 18 and $81 / 2$ in. long, respectively. A hand-operated guillotine door was placed between the start box exit and the start stem. When the start box was placed at the rear of the start stem (SBR) there was about 12 in. between the guillotine door and the center of the choice point. When the start box was placed forward (SBF) there remained only 3 in. between the start box guillotine door and the center of the choice point.

All rats were placed on an ad lib feeding schedule 10-14 days preceding the experiment. The animals were run under conditions of 20 to $21-\mathrm{h}$ food deprivation without reinforcement in a $\mathrm{T}$-maze which contained the start box at the north end of the start stem. Twenty-five $S$ s were randomly assigned to the SBR condition and the remaining 25 Ss to the SBF condition during Test Trial 1. Test Trial 2 consisted of the 25 SBR (SBF) Ss run under the SBF (SBR) conditions; Ss were tested in an alternate manner four times in the SBR and four times in the SBF conditions, generating a total of 200 possible alternation observations. The time between replications was about 45 min per $S$.

An alternation test consisted of placing the rat in the start box, allowing 2 min for the animal to circle $360 \mathrm{deg}$, and
While investigating the effects of discrimination seversal problems, $R$
Peckinpaugh, \& Stutz (1969) under moderate drive conditions. Douglas (1966) also demonstrated that the level of SAB was
Douglas (1966) found that satiated rats demonstrated spontaneous alternation behavior (SAB) to directional cues, a finding since replicated by Richman, Juels, and Tessnear observed that a rat's the animal's initial behavior in the start a clockwise (CW) fashion and the start box was located at the north end of the start turn east/left at the choice point. Likewise, animals that circled counterclockwise (CC) in the SB turned west/right at the choice was observed between the 